\title{
Co-infection with coxsackievirus A5 and norovirus GII.4 could have been the trigger of the first episode of severe acute encephalopathy in a six-year-old child with the intermittent form of maple syrup urine disease (MSUD)
}

Ákos Boros ${ }^{\mathrm{a}, \mathrm{b}}$, Péter Pankovics ${ }^{\mathrm{a}, \mathrm{b}}$, Sándor Kőmíves ${ }^{\mathrm{c}}$; Zoltán Liptai ${ }^{\mathrm{c}}$; Sarolta Dobner ${ }^{\mathrm{c}}$, Enikő Ujhelyi $^{\mathrm{c}}$, György Várallyay ${ }^{\mathrm{d}}$, Petra Zsidegh ${ }^{\mathrm{e}}$, Nóra Bolba ${ }^{\mathrm{a}, \mathrm{b}}$, Gábor Reuter $^{\mathrm{a}, \mathrm{b} *}$

${ }^{\text {a }}$ Regional Laboratory of Virology, National Reference Laboratory of Gastroenteric Viruses, ÁNTSZ Regional Institute of State Public Health Service, Pécs, Hungary

${ }^{\mathrm{b}}$ Department of Medical Microbiology and Immunology, University of Pécs, Pécs, Hungary

'Szent István and Szent László Hospital, Budapest, Hungary

${ }^{\mathrm{d}}$ Semmelweis University, Budapest, Hungary, MR Research Centre

${ }^{\text {e }}$ Semmelweis University, Budapest, Hungary, $1{ }^{\text {st }}$ Department of Paediatrics

Running title: Coxsackievirus A5 and norovirus co-infection triggered MSUD

* Address for correspondence:

Dr Gábor Reuter

Department of Medical Microbiology and Immunology

University of Pécs

H-7624 Szigeti út 12. Pécs, Hungary

Telephone: +36 (72) 536252

Fax: +36 (72) 536253

Email: reuter.gabor@gmail.com 


\begin{abstract}
In this case study, a coxsackievirus A5 (Picornaviridae) and a norovirus GII.4 (Caliciviridae) co-infection were detected using RT-PCR from a faecal sample of a 6-year-old girl with symptoms of severe acute encephalopathy subsequently diagnosed as intermittent form of maple syrup urine disease (MSUD). The two co-infecting viruses were hiding and triggering the underlying metabolic disorder. The genotyping of the viruses as well as the chronological course, wide spectra of laboratory tests and clinical presentations of this case which includes recurrent vomiting without diarrhoea, metabolic acidosis, unconsciousness, seizure and circulatory collapse, but with positive final outcome is also presented.
\end{abstract}


Maple syrup urine disease (MSUD) or branched-chain ketoaciduria is a rare but lifethreatening metabolic disorder of autosomal recessive inheritance named after the characteristic odour of affected infants' urine reminiscent of maple syrup [1]. The genetic background of MSUD contains multiple alterations in either branched chain $\alpha$-ketoacid dehydrogenase (BCKDH) A, B or dihydrolipoamide branched chain transacylase (DBT) E2 genes [2,3]. Mutational changes of $\mathrm{BCKDH}$ enzyme complex cause the elevation of the branched-chain amino acids (BCAA) including valine, isoleucine and leucine in the patient's sera and eventually lead to metabolic crisis [2,3]. Based on the clinical manifestations MSUD patients can be classified into classical or different, non-classical phenotypes called intermediate, intermittent, thiamine-responsive, and dihydrolipoyl dehydrogenase (E3)deficient $[2,3]$. The untreated newborns with classical MSUD - which is the most common type - show various symptoms of CNS involvement a few days after birth. Children with the intermittent form manifest no symptoms, have average level of plasma BCAAs and normal physical growth therefore the presence of MSUD could remain hidden even for long time. The onset of non-classical form of MSUD is suggested to connect to various causes of physiologic stress including common bacterial or viral infections which could trigger the degradation of muscle proteins rich in BCAA [4,5]. During the MSUD induced metabolic crisis symptoms may include vomiting, dehydration, ketoacidosis, ketonuria, lethargy, rapid neurological decline including seizures and encephalopathy [3]. These resemble the symptoms of encephalitis which include altered mental state (e.g. disorientation, speech disturbances), focal or diffuse neurological signs, seizures, fever, and imaging (e.g. magnetic resonance - MRI) and/or electroencephalogram (EEG) consistent with encephalitis. Other common clinical features like headache and bouts of vomiting are often present, as is cerebrospinal fluid (CSF) pleocytosis [6]. The etiological background of encephalitis could include c.a. 100 different pathogens; most of them are viruses like parechoviruses, 
enteroviruses (family Picornaviridae), herpesviruses (family Herpesviridae), arboviruses and a number of non-microbial processes like autoimmune (including acute disseminated encephalomyelitis - ADEM) and paraneoplastic encephalitis [6,7]. Furthermore most symptoms of metabolic crisis in MSUD can also be present in a severe, untreated gastroenteritis caused by e.g. noroviruses (NoV, family Caliciviridae), or rotaviruses (family Reoviridae). These symptoms are mostly due to the indirect effect (e.g. severe dehydration, electrolyte imbalance and acidosis) of the viral infection induced frequent diarrhoea and/or vomiting especially among children and the elderly [8-10]. The aim of this study was to show the clinical course of a 6-year-old patient with acute encephalopathy and metabolic crisis, subsequently diagnosed with intermittent form of MSUD, including the detailed chronological presentation of symptoms, diagnostic and therapeutic activities during the hospital stay. The patient had coxsackievirus A5 (family Picornaviridae) and norovirus GII.4 (family Caliciviridae) co-infection which triggered and masked, because of the overlapping clinical symptoms, the onset of an intermittent form of MSUD. They might have contributed to the severity of the metabolic crisis.

On July 29, 2015 a 6-year-old euthermic and conscious girl was presented at the emergency room of Szent János Hospital (Budapest) with severe dehydration, dizziness and slurred speech. The patient's symptoms started four days earlier with fever and vomiting but no diarrhoea (Fig. 1). Routine blood tests disclosed leukocytosis, hyponatraemia, slightly impaired renal function, metabolic acidosis and increased anion gap (Fig. 1). Parenteral fluid administration resulted in temporary improvement; however, the next morning she became unconscious and developed a seizure successfully stopped by benzodiazepine. Despite the use of furosemide and mannitol she remained unconscious. Mechanical ventilation was started, and the patient was transferred to the Pediatric Intensive Care Unit of Szent István and Szent László Hospital (Budapest) for suspected encephalitis. Intravenous acyclovir and ceftriaxone 
were initiated. CSF protein and glucose levels and cell count were normal. Nasal, throat, tracheal swabs, urine, CSF and blood samples were taken for bacteriological and mycological diagnostics, but microbial pathogens were not detected. A mild circulatory impairment was stabilized by the administration of fluid, albumin and inotropic medication. Results of the blood test were returned to normal (Fig. 1). Upon neurological testing the pupils were wide but appropriately reacted to light, there was no optic disc congestion, and the child had no focal deficit. EEG showed severe diffuse dysfunction. ADEM could not be excluded and methylprednisolone was started. Cranial MRI showed symmetrical lesions of the brainstem, the thalami, the cortex and the cerebellar dentate nuclei (Fig. 2). This level of symmetry is uncommon in ADEM. Repeated MRI revealed regression of the lesions radiologically interpreted as of either viral, toxic or metabolic origin. Mass lesions, haemorrhage and ischemia could be excluded. As the disease seemed to be infection-related, faecal, serum, cerebrospinal fluid (CSF) and nasopharyngeal aspirate samples were collected for diagnostic purposes. Pathogenic microbes were not detected by bacteriological and mycological tests and cultures. Autoimmune encephalitis auto-antibodies against glutamate receptors (NMDA, AMPA1, AMPA2), CASPR2, LGI1, GABARB1/B2 and anti-aquaporin-4 antibodies were negative in serum and CSF. Adenovirus, HHV1/2 and Varicella-Zoster virus (VZV) DNAs were not detectable by PCR in either serum or CSF samples. Antibodies against tick-borne encephalitis virus and West Nile virus were not shown in the analyzed samples as well. Total RNA was extracted from the faecal, serum and cerebrospinal fluid (CSF) samples using TRI reagent ${ }^{\circledR}$ (MRC, USA) according to the manufacturer's instructions. The presence of enterovirus and parechovirus was tested using RT-PCR and generic primer pairs targeting the 5'UTR regions (enteroviruses: UnivEnt-5UTR-R: 5'-ATTGTCACCATAAGCAGCCA-3' and UnivEnt-5UTR-F: 5'-GTACCYTTGTRCGCCTGTT-3'; parechoviruses: HPeV-5UTRRgen: 5'-CCAGATCAGATCCATAGTGTC-3' and HPeV-5UTR-Fgen: 5'- 
GATGGCGTGCCATAAYTCTA-3'. All specimens were negative for human parechovirus, but the faecal sample showed enterovirus positivity. Furthermore, a critical revision of the patient's history with several bouts of vomiting raised the suspicion of gastrointestinal (GI) viral infection; therefore faecal sample was tested retrospectively for rotavirus/adenovirus using immunochromatographic assay (CerTest-Biotec, Zaragoza, Spain) and for norovirus by RT-PCR using the generic primer pairs of JV12Y (5'-ATACCACTATGATGCAGAYTA-3) and JV13I (5'-TCATCATCACCATAGAAIGAG-3') targeting the RNA polymerase region [11]. The faecal sample was negative for rotavirus and adenovirus but positive for norovirus. Norovirus RNA was not detected in serum and CSF samples. The viral load of the detected enterovirus $(2.36 \mathrm{E}+06 / \mathrm{mL}$ faeces $)$ and norovirus $(4.5 \mathrm{E}+07 / \mathrm{mL}$ faeces $)$ were determined using SYBR Green-based real-time PCR assay (Maxima SYBR Green qPCR Master Mix, Thermo Scientific, USA). For the generation of standard curves ten-fold dilution series of silicacolumn (Qiagen, Germany) purified and spectrophotometrically quantified RT-PCR amplicons of detected norovirus and enterovirus were used. These results suggest that norovirus and enterovirus co-infection was present during the ongoing severe encephalopathy. In the next few days there was a continuous improvement of the patient's neurological condition. Her consciousness returned. The control EEG showed only mild alteration. The patient was weaned from ventilator and discharged from hospital 14 days after admission without neurological sequelae. Two and a half months later this girl was hospitalized again for acute onset of vomiting, ataxia, lethargy and disturbed consciousness in the context of a viral illness. Laboratory testing revealed abnormalities similar to those during the first episode. The recurrence of encephalopathy, acidosis and increased anion gap, the symmetrical MRI pattern made a metabolic disorder most likely. Tandem mass spectrometry (MS) was performed and detected significant elevation in serum levels of branched chain amino acids, especially of leucine. DNA sequencing of the BCKDH-B gene found a potential compound heterozygous 
state for a known pathogenic variant (c.832G $>$ A) on one allele and a likely pathogenic, so far not reported frameshift mutation (c.887_896dup) on the other, in accordance with the biochemical diagnosis of maple syrup urine disease (MSUD). The normal motor and cognitive development with episodes of encephalopathy during febrile infections is typical of the intermittent form of the disease. The child did not have further recurrences during the past 18 months and her leucine, isoleucine and valine levels remained normal on a diet with restricted meat consumption. For the reliable typing the full-length genome of the detected enterovirus strain as well as the full capsid sequence of the detected norovirus strain were determined using RT-PCR and 5'/3' RACE PCR methods with sequence-specific primer pairs $[12,13]$. The PCR products were sequenced directly in both directions with the BigDye Terminator Cycle Sequencing Kit (Applied Biosystems, Warrington, UK) and run on an automated sequencer (ABI PRISM 310; Applied Biosystems). The determined genomic sequences were deposited in the GenBank database under accession numbers of KU761262 and KY341923. The sequence alignments and identity calculations were performed with the BioEdit software (version 7.1.3.0) using the ClustalW algorithm. The bootstrapped (1000 replicates) neighbour joining method with the Jukes-Cantor matrix-based model was used to construct the VP1 phylogenetic tree of enterovirus nucleotide sequences by MEGA software ver. 6.06 [14]. The 7405-nt-long genome of CVA5/13164/HUN/2015 [KU761262] follows the general enterovirus genome layout and shows $92.5 / 98.3 \%, 92.7 / 98.6 \%$ and $92.5 / 98.7 \%$ nt/aa identities to the $\mathrm{P} 1, \mathrm{P} 2$ and $\mathrm{P} 3$ genome regions of coxsackievirus A5 strain CVA5/SD/CHN/09 (HQ728261) as the closest match identified by BLAST search. Based on the high sequence identity values CVA5/13164/HUN/2015 most likely belongs to the CV-A5 genotype. Because of the small number of complete $\mathrm{CV}-\mathrm{A} 5$ genomes $(\mathrm{N}=5)$ available to date the phylogenetic analysis of the study strain was restricted to the 314-nt-long partial VP1 sequences of CV-A5 from which numerous sequences were available in the GenBank 
database. In the VP1 phylogenetic tree the CVA5/13164/HUN/2015 is clustered together with several CV-A5 strains collected after 2006 with the closest phylogenetic relationship to strain NIV093891LV17 (JX476134) which was identified from a faecal sample originated from a patient with acute flaccid paralysis in India [15] (Fig. 3). These two sequences share $95.8 / 100 \% \mathrm{nt} / \mathrm{aa}$ identity to each other. Based on the 3207-nt-long partial polymerase and complete capsid sequence, the detected norovirus strain $\mathrm{Hu} / \mathrm{GII} .4 / 13164 / 2015 / \mathrm{HUN}$ [KY341923] belongs to the genogroup II (GII) with the closest sequence relative of GII.4 genotype of norovirus strain Hu/GII.4/Sydney/NSW0514/2012/AU (JX459908) using the Norovirus Genotyping Tool Ver. 1.0 [16]. These two sequences share 98\% nt identity at the corresponding regions.

In this study, a coxsackievirus A5 and a norovirus GII.4 co-infection were detected from a faecal sample of a 6-year-old girl with symptoms of severe acute encephalopathy which was subsequently diagnosed as an intermittent form of maple syrup urine disease (MSUD). Furthermore the first complete genome of coxsackievirus A5 from Hungary was determined and characterized which is also the first complete CV-A5 genome originated from Europe. The identified norovirus strain belongs to the GII genogroup and shows closest relationship to a GII.4 genotype which was the predominant norovirus genotype circulating worldwide including Hungary in 2015 and could be associated with more severe outcomes than non-GII.4 strains [10]. Although the decisive diagnosis of MSUD (detection of high level of BCAAs in the patient's serum by tandem MS) was conducted at the onset of the second episode, the presence of known and novel, likely pathogenic mutations in the BCKDH-B gene suggested that the first episode was also due to MSUD. This is supported by the observation that episodes of the intermittent form of MSUD are usually related to massive physiologic stress often triggered by bacterial or viral infection [4,5]. In our case this definitive trigger was most likely a NoV and CV-A5 co-infection. The non-classical types of MSUD usually 
have their onset by the age of two years [4,5], but in the presented case MSUD remained hidden until the patient's age of 6. This was most likely due to the lack of severe physiological stress until the co-infection caused by CV-A5 and NoV GII.4. The severity of the CV-A5 / NoV co-infection was suggested by the relatively high viral load found in the patent's faeces collected a week after the onset of the GI symptoms. Usually the early diagnosis of late onset of non-classical MSUD is initially started by the recognition of atypical courses of common infections [5]. In cases where symptoms of the ongoing infection(s) and those of MSUD overlap, diagnosis could be incorrect and MSUD may remain undetected even in spite of typical symptoms. In the presented case, the symptoms accompanying the viral co-infection of CV-A5 and NoV-GII.4 could just as easily indicate the onset of an MSUD- related metabolic crisis [3]. Therefore these symptoms mimicked those of infection-related encephalitis, and only their reappearance at the second hospitalization turned attention to metabolic disorders. On metabolic screening MSUD was diagnosed and this was supported by the positive finding of DNA analysis. This intermittent form manifests with recurrent bouts of encephalopathy most likely triggered first by prior infections of CV-A5/NoV-GII.4.

The CNS symptoms as well as the repeated bouts of vomiting without diarrhoea diverted the attention from a potential gastrointestinal disease therefore the NoV-infection was diagnosed later, after the detection of CV-A5. The lack of diarrhoea is common in norovirus-related gastroenteritis of children therefore in case of episodes of vomiting even without diarrhoea a possible norovirus infection should be kept in mind [10, 17].

Though tandem mass spectrometry (MS/MS)-based amino acid profiling of serum and molecular detection of pathogenic variants of BCKDHA/B and DBT genes associated with MSUD are not part of routine diagnostics, in case of severe metabolic acidosis and CNS involvement the isoleucine and leucine levels should be tested regardless of the presence of 
gastrointestinal infection [3]. We think this case study is a good example of multidisciplinary effort and continuous real-time revision of clinical thinking in a severe, life-threatening disease, where both the trigger and the underlying were finally identified.

\section{Acknowledgements}

This work was supported by grant from the Hungarian Scientific Research Fund (OTKA/NKFIH K111615). Á.B. and P.P. were supported by the János Bolyai Research Scholarship of the Hungarian Academy of Sciences.

\section{Compliance with Ethical Standards}

Funding: This study funded by Hungarian Scientific Research Fund (OTKA/NKFIH K111615)

Conflict of interest: The authors declare that they have no conflict of interest.

Ethical approval: All procedures performed in studies involving human participants were in accordance with the ethical standards of the institutional and/or national research committee and with the 1964 Helsinki declaration and its later amendments or comparable ethical standards. This article does not contain any studies with human participants performed by any of the authors.

Informed consent: Informed consent was obtained from all individual participants included in the study. 


\section{References}

1. Menkes JH, Hurst PL, Craig JM (1954) A new syndrome: progressive familial infantile cerebral dysfunction associated with an unusual urinary substance. Pediatrics. 14:462-467.

2. Chuang DT, Shih VE, Wynn RM (2001) Chapter 87: Maple syrup urine disease (branchedchain ketoaciduria). In: The Metabolic and Molecular Basis of Inherited Disease. McGrawHill, S. CR, New York, pp. 1971-2006.

3. Strauss KA, Puffenberger EG, Morton DH (2013) Maple Syrup Urine Disease. In: Pagon RA, Adam MP, Ardinger HH, Wallace SE, Amemiya A, Bean LJH, Bird TD, Fong CT, Mefford HC, Smith RJH, Stephens K, editors. GeneReviews. Seattle (WA): University of Washington, Seattle; 1993-2016. PMID: 20301495

4. Asola MR (1995) A diver unconscious after gastroenteritis. Lancet. 18:1338.

5. Axler O, Holmquist P (2014) Intermittent maple syrup urine disease: two case reports. Pediatrics. 133:e458-60.

6. Piquet AL, Cho TA (2016) The Clinical Approach to Encephalitis. Curr Neurol Neurosci Rep. 16:45.

7. Pallansch MA, Roos RP (2001) Enteroviruses: polioviruses, coxsackieviruses, echoviruses, and newer enteroviruses. In Fields Virology, 4th edn, pp. 723-775. Edited by D. M. Knipe \& P. M. Howley. Philadelphia: Lippincott Williams \& Wilkins.

8. Chen SY, Tsai CN, Lai MW, Chen CY, Lin KL, Lin TY, Chiu CH (2009) Norovirus infection as a cause of diarrhea-associated benign infantile seizures. Clin Inf Dis 48: 849-855. 9. Morita T, Fujieda M (2011) Acidosis with hyperuricemia and renal tubular damage in viral gastroenteritis. Ped Nephrol, 26: 2259-2260.

10. Robilotti E, Deresinski S, Pinsky BA (2015) Norovirus. Clin Microbiol Rev 28:134 -164. 
11. Vennema H, de Bruin E, Koopmans M (2002) Rational optimization of generic primers used for Norwalk-like virus detection by reverse transcriptase polymerase chain reaction. $\mathrm{J}$ Clin Virol 25: 233-235.

12. Boros A, Pankovics P, Simmonds P, Reuter G (2011) Novel positive-sense, singlestranded RNA (+ssRNA) virus with di-cistronic genome from intestinal content of freshwater carp (Cyprinus carpio). PLoS One 6, e29145.

13. Boros A, Pankovics P, Knowles NJ, Reuter G (2012) Natural interspecies recombinant bovine/porcine enterovirus in sheep. J Gen Virol 93:1941-1951.

14. Tamura K, Stecher G, Peterson D, Filipski A, Kumar S (2013) MEGA6: Molecular Evolutionary Genetics Analysis version 6.0. Mol Biol Evol 30: 2725-2729

15. Laxmivandana R, Yergolkar P, Gopalkrishna V, Chitambar SD (2013) Characterization of the non-polio enterovirus infections associated with acute flaccid paralysis in South-Western India. PLoS One. 22:e61650.

16. Kroneman A, Vennema H, Deforche K, Avoort HV, Peñaranda S, Oberste MS, Vinjé J, Koopmans (2011) An automated genotyping tool for enteroviruses and noroviruses. J Clin Virol. 51:121-125.

17. Reuter G, Farkas T, Berke T, Jiang X, Matson DO, Szücs G. (2002) Molecular epidemiology of human calicivirus gastroenteritis outbreaks in Hungary, 1998 to 2000. J Med Virol. 68:390-398. 


\section{Figure legends}

Fig. 1. Chronological summary of the clinical case. The horizontal black line with numbers represents days from July 25 to Aug 11, 2015. The symptoms (blocks with gray background) and actions (white blocks) are depicted under the date line. Horizontal grey arrows represent the durations of the drug treatments. The results of laboratory tests and clinical examinations are shown under the date line. Detailed discussion of the tests and examinations are found in the text. Abbreviations: n.d.: not determined, WBC: white blood cells, NEU: neutrophil granulocytes, MRS: magnetic resonance spectroscopy, CRP: C-reactive protein, Na: Sodium, KN: urea, bact. \& mycol.: bacteriological and mycological, Virol. tests: virological tests, Prot.: Protein (unit: g/L) Glu: glucose (unit: mmol/L). PICU: Pediatric Intensive Care Unit, LP: lumbar puncture, CV-A5: coxsackievirus A5, NoV: norovirus.

Fig. 2. Axial FLAIR (A-D) and diffusion-weighted images (DWI: E-H) of the patient show symmetrical areas of increased signal (arrows) involving the cerebral cortex (A), the thalami (B), the midbrain (C), the pons and the cerebellar dentate nuclei (D). Some of these lesions manifest restricted diffusion (high signal on DWIs: E, F and G).

Fig. 3. Phylogenetic relationship of CVA5/13164/HUN/2015 (in bold and marked with an arrow) and the closest relatives of CV-A5 sequences (strain names followed by the accession numbers in square brackets, the country and year of isolation) based on the Neighbor-Joining tree of 314-nt-long partial VP1 sequences. CV-A5 strains with available complete genomes are underlined and marked with asterisk. Coxsackievirus A12 (CV-A12) strains were used as outgroup. Note that the CV-A5 strains identified after $2006(\geq 2006)$ are clearly separated from strains isolated earlier $(\leq 2003)$. 
Figure 1.:

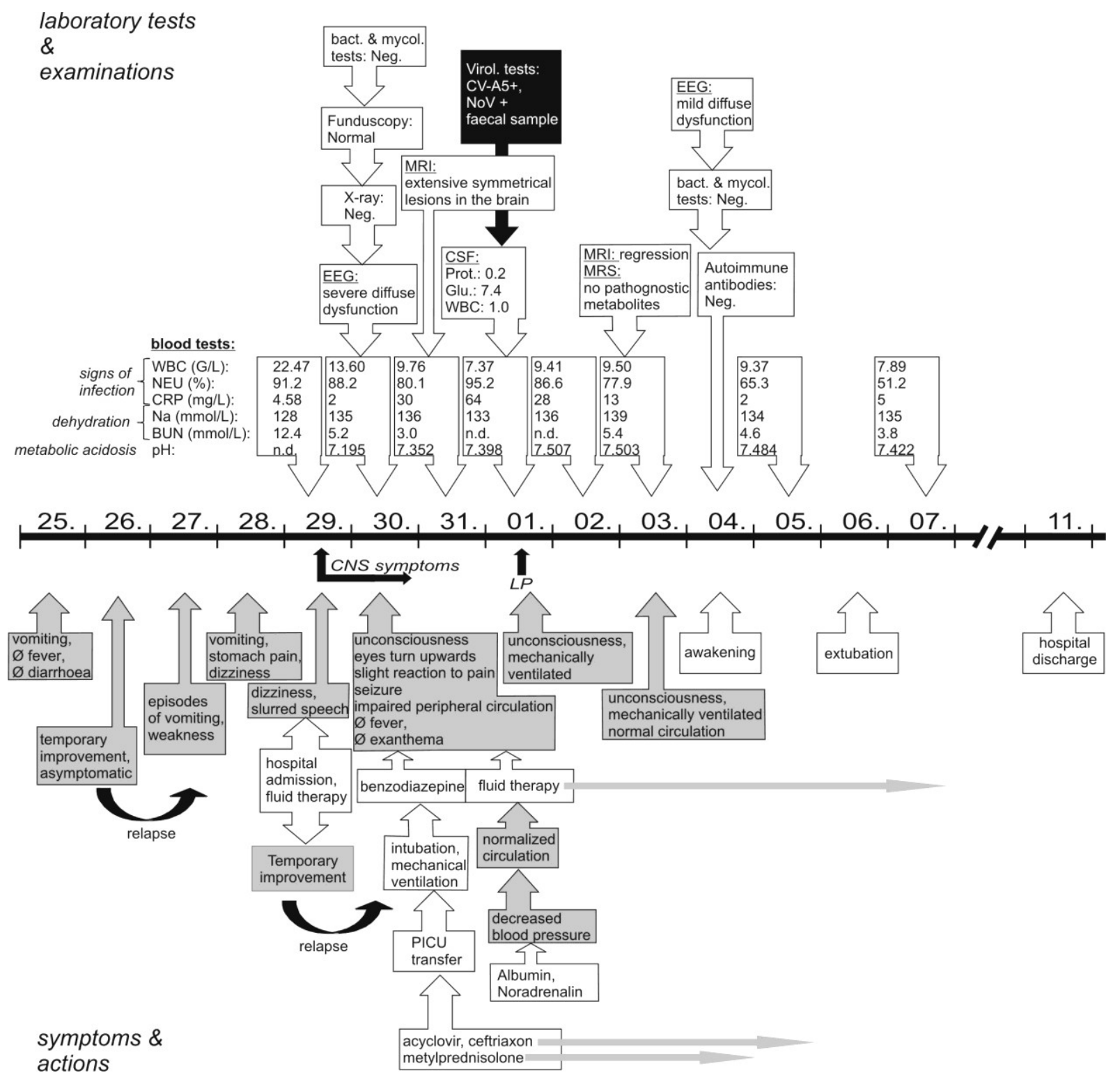


Figure 2.:

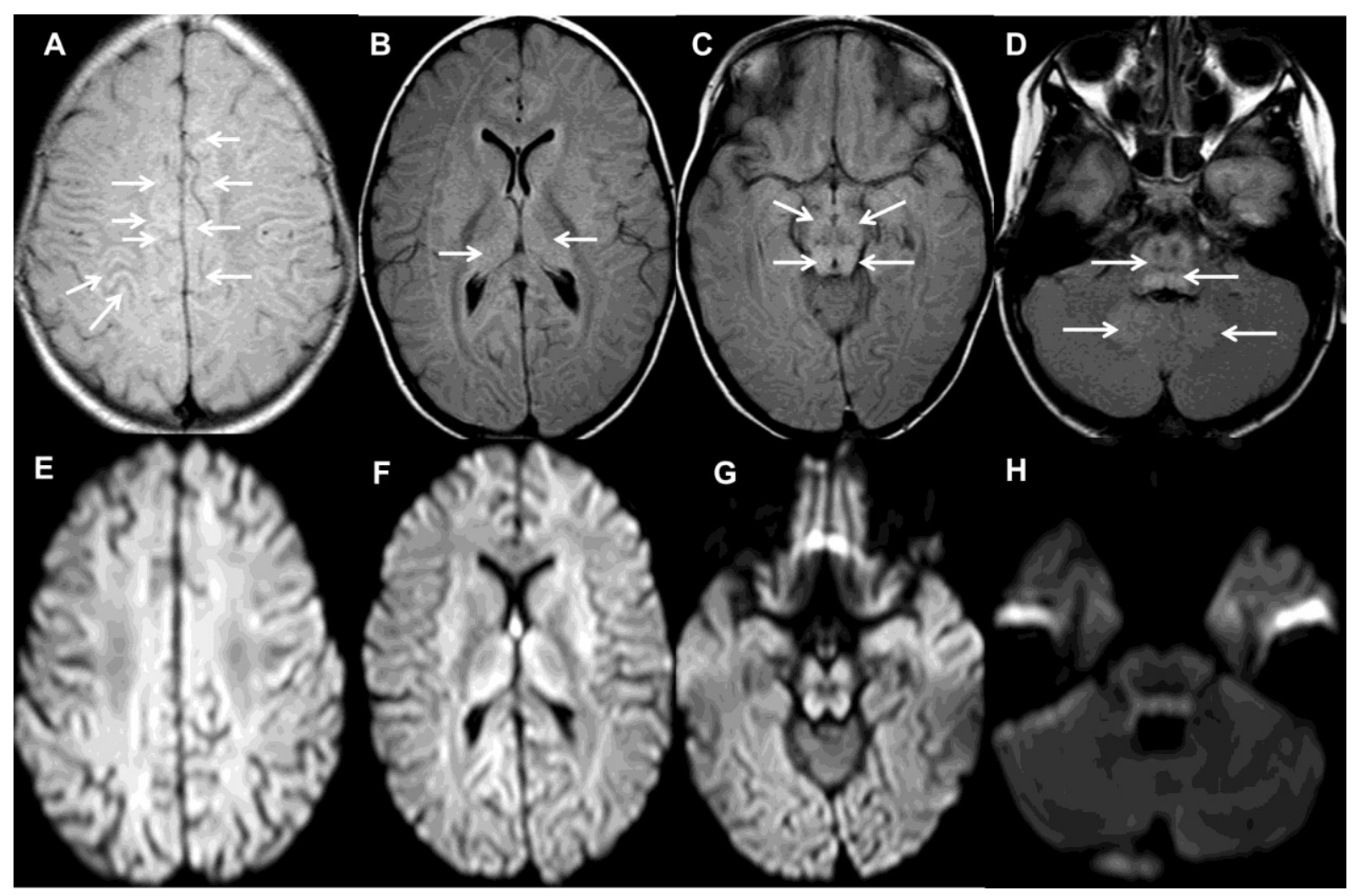


Figure 3.:

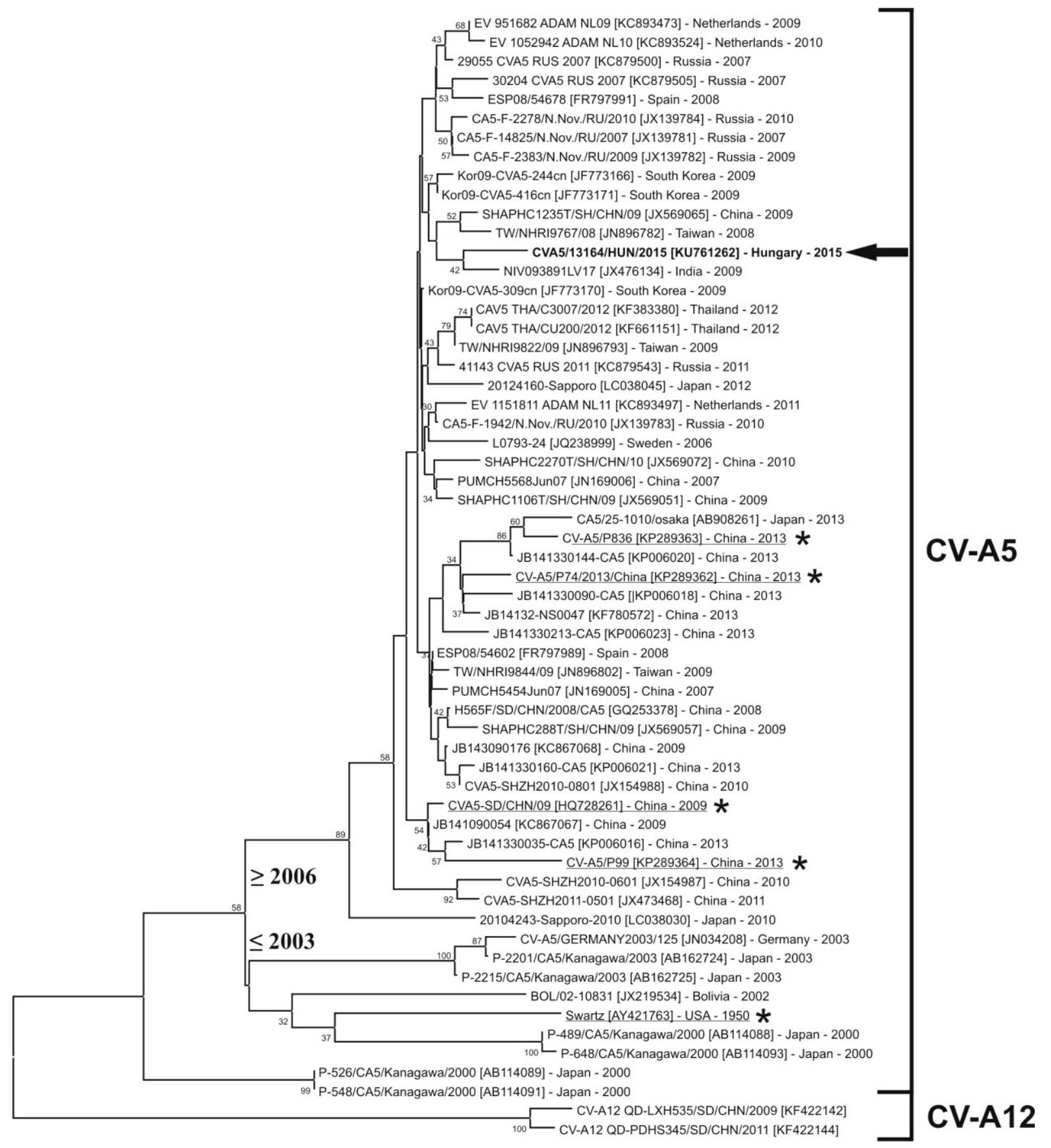

